\title{
El rol de las estatalidades en la construcción de sujetos asociados a la gestión hídrica del Chile reciente
}

\author{
Felipe Trujillo Bilbao ${ }^{1}$ \\ Recibido: 14 de diciembre de 2018 - Aprobado: 01 de marzo de 2019
}

\begin{abstract}
Resumen
La historia ambiental es entendida aquí como una invitación a observar en la naturaleza indicios de las transformaciones sociotécnicas del Chile contemporáneo. Se revisan los principales hallazgos de la producción historiográfica y científico-social actual sobre la gestión de los diversos tipos de agua en Chile. Entendida ésta en su condición de material, política y biopolítica, se da cuenta de cómo ha transitado por tres estatalidades orquestadas paralelamente por distintos órganos del Estado chileno: un movimiento constante de zigzag entre la conservación, la desregulación y la tecnificación. Se defiende la propuesta de que, en el entrecruce entre medioambiente y política, está la clave para interrogar, de manera histórica, a la gestión hídrica como decidora de una serie de problemas directamente vinculados a la construcción del Chile contemporáneo y no como relegada a otros problemas clásicos, como la Reforma Agraria, para la segunda mitad del siglo XX, o los agronegocios, para fines del siglo XX, sin desconocer por ello el rol que estos procesos han tenido en la gestión hídrica del país.
\end{abstract}

Palabras clave: gestión hídrica, estatalidades, neoliberalización, Chile contemporáneo.

\section{The Role of State Policy in the Construction of Subjects Associated with Water Management in Contemporary Chile Abstract \\ Environmental history is understood here as an invitation to observe in nature indications of the sociotechnical transformations of contemporary}

Chileno. Antropólogo y Magíster (c) en Historia del Chile Contemporáneo, Universidad Alberto Hurtado, Chile. Dirección de Investigación y Postgrados, Facultad de Filosofía y Humanidades, Universidad Alberto Hurtado. El presente estudio forma parte de la investigación de tesis de magíster del autor, desarrollada con apoyo del Programa de Formación de Capital Humano Avanzado, CONICYT, Becas Magíster Nacional F22181741. E-mail: ftrujillo@uahurtado.cl 
Chile. This article looks at the main findings of current historiographic and scientific-social production on the management of different types of water in Chile. Understanding water as a political and biopolitical material, this paper looks at how it has been subject to three state policies orchestrated in parallel by different bodies of the Chilean Government: a constant zigzag movement between conservation, deregulation and technification. It defends the proposal that at the intersection of the environment and politics is the key to a historical inquiry into water management as a deciding factor for a number of problems directly related to the construction of contemporary Chile, and not relegated to other classical problems like the Agrarian Reform of the second half of the $20^{\text {th }}$ century or agribusiness at the end of the $20^{\text {th }}$ century, without fully disregarding the role that these processes have played in the country's water management.

Keywords: Water management, statehoods, neo-liberalization, contemporary Chile.

Este artículo busca interrogar, a la luz de la producción historiográfica disponible, cómo se ha producido una estatalidad en Chile vinculada a la gestión y distribución del agua. El sentido de ello es observar el panorama de enfoques y acciones que son ejecutadas en el Chile contemporáneo sobre lo ambiental, y el rol que sus transformaciones juegan en la construcción del proyecto neoliberal de país posdictadura.

En este contexto, se propone interrogar a las transformaciones socioproductivas posibilitadas por el agua a partir de un enfoque de la estatalidad, entendiendo que de ella depende en gran medida la producción de agentes que se vinculan con el agua en sus múltiples dimensiones, siguiendo la matriz propuesta por Bakker (2012), a saber, comprender al agua como un elemento que permite movilizarse entre lo político, lo biopolítico, gestión y control de los elementos que posibilitan la vida y lo material. ${ }^{2}$

Es así como el agua clarifica otros procesos vinculados a la construcción política del Chile reciente, no solo porque la forma en que ella se transa o se conserva es sintomática de las políticas neoliberales sobre la naturaleza (Bauer, 2002), sino porque además es una plataforma para observar cuáles son los puentes tendidos para la continuidad sociopolítica entre dictadura militar y transición democrática a fines del siglo XX. De esta manera, es posible

2 Particular interés toma en esta apuesta la relación entre agua y ruralidad. No obstante, cabe advertir, se espera también escapar -y en el proceso explicar su sentido- a la sinonimia temprana que se hace en la investigación social entre agua y campesinado. Esto quiere decir que una revisión al agua como ligada a las actividades agrícolas propiamente tales supone una limitación de la mirada y constriñe las múltiples dimensiones del agua como plataforma para observar las transformaciones del Chile contemporáneo. 
prestar atención a los roles de diversos agentes en la disputa por el poder, por la producción de los paisajes del Chile contemporáneo y los sujetos y escenarios productivos en que ellos se desenvuelven.

La relación entre agua y poder, por lo tanto, constituye un punto clave para observar procesos que no solamente se remiten a las transformaciones productivas en la gestión hídrica o las actividades de agricultura o de extracción hidrodependientes, sino que además pueden comprenderse como un nexo para reflexionar sobre la producción de estatalidad. Sobre ello arroja luces el trabajo de Swyngedouw (2007) que desde el agua -o el hidropoder, en sus palabras-, reconstruye la España franquista, como también puede observarse en la obra de Pflieger (2008) sobre cómo la canalización en Chile durante el siglo XX fue un elemento determinante de la segregación socioespacial que ha caracterizado a Santiago.

Al proveer una mirada comparada y continental sobre el agua en el siglo XX, llama la atención la revisión y procedimiento seguido sobre el caso mexicano por parte de Tortolero (2000), quien ilustra la transición desde bienes libres hacia bienes económicos, detonando problemas de escasez y distribución desigual. No solo ello, a pesar de identificar que el problema del agua es más bien interdisciplinar, identifica en la Historia una capacidad de mayor rendimiento para una lectura que es simultáneamente material, cultural y económica.

Es por ello que, como objeto de investigación histórica, el agua y su gestión en Chile han suscitado un interés para académicos de otras latitudes, levantando otro aspecto prolífero para el desarrollo de una perspectiva chilenista de las transformaciones neoliberales que construyen al Chile contemporáneo (Bauer, 2002). En suma, a partir del estudio de su historicidad, siguiendo a Gaynor, el presente texto busca reconstruir la dimensión política, biopolítica y material de la gestión del agua en Chile

Esta discusión es particularmente fértil en América Latina, donde la reflexión sobre proyectos pro Estado y la relación que éstos tienen para con el resguardo de recursos naturales estratégicos ha capturado un gran interés, tanto en la academia como en organismos internacionales. Es de esta manera como aparece, por ejemplo, en una detallada revisión realizada por Sojo (2011) a los tipos de estatalidad, como una rejilla conceptual que permite hacer un balance sobre las funciones y capacidades que determinada estatalidad puede ejercer en un contexto histórico y geográfico específico.

Para Sojo (2011), entonces, la estatalidad permite observar el comportamiento del Estado en una clave plástica y no ahistórica, toda vez que su campo de acción está íntimamente vinculado a elementos contextuales que pueden debilitar o fortalecer la institucionalidad, así como un umbral de 
políticas sociales, implementadas y por implementar, que van dando forma a las prácticas cotidianas de ejercer el Estado, y que construyen ilustradores perfiles de desarrollo de Estado, gobernabilidad y capacidades por desplegar en la activa construcción de un proyecto social y económico (Sojo, 2011: 39). Este tipo de evaluación, argumenta Sojo, toma sentido para Latinoamérica, particularmente toda vez que la estatalidad desplegada aparece como un aspecto sintomático de las diversas estrategias de consolidación de la democracia electoral en el continente.

Estatalidad que, por cierto, está en constante movimiento y disputa. Una serie de elementos nacionales e internacionales modelan a la estatalidad y a la percepción que de ella es levantada por la ciudadanía. Esto se evidencia al observar cómo, entre 1930 y 1980, Latinoamérica presenta vertiginosas modificaciones tanto en la regularidad de sus cambios de gobierno, la presencia de golpes de Estado como también, con mayor notoriedad a fines del siglo XX, una democratización de la participación política y representación parlamentaria en grupos históricamente excluidos (Programa de las Naciones Unidas para el Medio Ambiente, 2000).

En un nivel conceptual, la estatalidad ha sido revisada como patrimonio compartido de las ciencias sociales, y con especial utilidad para el ejercicio comparativo y de evaluación sobre el despliegue, recepción y capacidad de ciertos elementos de la matriz política por legitimarse o transformar lo social. De acuerdo con O'Donnell (2010), se basa en una idea del Estado como una entidad regulatoria multidimensional, que modela territorios, fronteras, actitudes y reglas con las cuales corporaciones, individuos y otros sujetos colectivos se desplieguen cotidianamente.

En ese sentido, la noción de estatalidad se puede comprender como la manera en que se materializa una forma de hacer Estado en determinados sujetos, agentes o actividades (Cowan, 2016). En términos historiográficos, su rendimiento ha estado principalmente asociado con la capacidad explicativa de revisitar elementos constitutivos de la forma de ejercer Estado en ciertos países, predominantemente latinoamericanos, y en base a ello indagar cómo fueron tomando forma ciertos elementos constitutivos, como la desposesión indígena en países con historias de violentos procesos de unificación en contra de territorialidades precolombinas (Castro y Lavinas, 2017).

La delimitación propuesta en esta investigación apunta a revisar la producción historiográfica y científico-social en torno a la historia de la gestión del agua en Chile para la segunda mitad del siglo XX. Con base en esos hallazgos, en un segundo momento del texto se propone una tipología de estatalidades presentes de manera interrelacionada, que están orientadas a promover un tipo de uso de las aguas, pero también un espíritu nacional de 
resguardo, consumo o contemplación del agua. En el proceso se identifican, además, cuáles son los órganos que el Estado dispone para desplegar dicha estatalidad.

Se procede primero interrogando al mismo campo disciplinar para encontrar en esta revisión teórica las pistas sobre el rendimiento investigativo de la propuesta, finalizando con un breve sumario de las estatalidades identificadas en la literatura y qué campos de investigación sobre lo contemporáneo son desde ellas abiertos.

Respecto de la estrategia metodológica que nutre al texto, ella se compone de información recopilada en literatura especializada, obtenida en una serie de bases de datos de revistas científicas indexadas del área de la historia y estudios urbanos, así como de revisiones históricas o de prensa elaboradas por los mismos organismos estatales que se han abocado a la gestión del agua. Esto tiene sentido toda vez que, como bien ha mostrado una serie de estudios sobre trayectorias institucionales y profesionales, son los mismos protagonistas del Estado quienes construyen activamente su memoria y los documentos con cargo a los cuales esta será visitada.

Asociado a lo anterior, se incorporan figuras y cuadros resúmenes obtenidos a partir de otras publicaciones que sirven para graficar uno de los puentes discursivos más prominentes en la historia de la gestión del agua en Chile: la justificación de su tecnificación y la sinonimia de ella con un tránsito hacia el desarrollo del país. En lo que sigue, entonces, se articulan estas reflexiones con la recopilación de información y análisis de datos disponibles en los trabajos académicos revisados para este artículo.

\section{La historia política y ambiental en torno a la gestión del agua}

"Encontrar el pasado en la naturaleza", es la invitación hecha en un volumen sobre historia ambiental publicado a fines del siglo XX (Myllyntaus y Saikku, 1999). Efectivamente, como el texto ensaya, hace falta explicitar las relaciones entre el medio ambiente y los procesos históricos. No es tanto una historización de las transformaciones en la biomasa -o de la disponibilidad de agua, en este caso-, sino una lectura a partir de estos elementos de fenómenos políticos que logran, desde la estatalidad y sus posibilidades, manifestarse en los paisajes, transformando y produciendo discursividades y regímenes emocionales inclusive. ${ }^{3}$ Así, el medioambiente, como concepto

Véase, por ejemplo, a modo general y dando luces metodológicas, el trabajo de Monique Scheer (2012), y también para un caso de estudio sobre las transformaciones ambientales 
más amplio que engloba lo referido al agua y su contacto con el paisaje, se presenta como una plataforma rica en interpretaciones y cruces posibles para la historia, pues permite acompasar a la naturaleza con los cambios en las relaciones humanas a través del tiempo.

Se subrayan a continuación tres caminos principales mediante los cuales historia y medioambiente pueden arrojar luces sobre problemas históricos contemporáneos, principalmente vinculados a la explicitación de los mecanismos en torno a los cuales reposa el proceso de neoliberalización en Chile (Gárate, 2012), explicitando junto a ello cuáles son las herramientas metodológicas con las cuales es posible sortear las dificultades que este tipo de problemas de investigación supone.

En primer lugar, la historia medioambiental permite proceder considerando a la subjetividad mediante herramientas de historia oral, con tal de acceder a los relatos y construcciones discursivas sobre la relación entre naturaleza y vida cotidiana. Como McCann y Gaynor (2017) muestran, el medioambiente evoca reflexiones de riqueza historiográfica, tanto sobre actividades productivas asociadas a la naturaleza, las éticas y discursos que sobre el medioambiente se sedimentan, pero también sobre cómo se llevan a cabo proyectos vitales insertos en una temporalidad, espacialidad y emocionalidad específica de la mano de ciertos territorios y paisajes.

En segundo lugar, las transformaciones ambientales promovidas por causas antrópicas traen consigo información sobre desarrollo tecnológico, organización de los emplazamientos habitacionales, desde comunidades indígenas a metrópolis, y proyectos urbanísticos a gran escala, detonados a partir de las soluciones planteadas -ya sea desde la estatalidad o a nivel más local- a dificultades de acceso, gestión y manejo de determinados recursos naturales. Sobre ello, Gaynor (2017a) observa cómo la escasez hídrica en zonas urbanas se relaciona con dinámicas y discursos asociados a la higiene, la emocionalidad de la vida acomodada y la domesticidad de la producción de jardines y patios.

En tercer lugar, en el medioambiente se puede observar, además, los rastros de transformaciones paisajísticas que son a su vez contraportada de proyectos productivos, creando "regiones productivas" o "regiones commodity", que son posibilitadas y legitimadas históricamente. En esta línea, vale la pena relevar el trabajo de Gaynor (2017b) sobre paisajes coníferos, como también la investigación de Bustos e Irarrázaval (2016) sobre regiones del salmón en el sur de Chile.

del Chile contemporáneo y su impacto en las emociones y el habitar, el trabajo de Skewes et al. (2017). 
Estas aproximaciones mencionadas son parte de una agenda de investigación mayor que coincide en valorar en los recursos naturales una puerta de entrada a transformaciones de diversa envergadura que se despliegan simultáneamente en diferentes escalas. Como tal, permitirá también detectar cómo se moviliza el Estado y sus agentes como activos productores de determinados regímenes de naturaleza. ${ }^{4}$

En Chile, particularmente, la Historia Ambiental ha permitido la producción de informados y problematizadores textos históricos y de teoría social sobre cómo se despliega la modernidad dependiente de la extracción de recursos naturales que son estratégicos en el territorio nacional. Así, por ejemplo, es concebido el trabajo de Camus (2005) sobre la gestión de los bosques en Chile, donde se puede observar no solo la disminución de biomasa vegetal en el país, sino cómo esta es sintomática del despliegue de nuevas relaciones de capital internacional y legislaciones permisivas de fomento forestal. Es más, el análisis de la gestión de los bosques en Chile arroja luces no solo sobre las consecuencias de la neoliberalización de la naturaleza en los recursos, sino que, además, en cómo se transforma dinámicamente la comprensión social de qué es la naturaleza y a qué propósitos sirve.

Como señalan Camus y Hajek (1998), tal y como se ha hecho mención aquí, es de hecho la interrelación de procesos productivos, recursos naturales y transformaciones societales lo que constituyen el enclave de interés:

"(el objetivo de la obra es) exponer un breve contexto económico y los efectos que han tenido las políticas económicas sobre los recursos naturales (forestales, pesqueros, mineros), los procesos y políticas de urbanización, y la visión global, incluyendo perspectivas científicas, técnicas, ciudadanas y académicas" (Camus y Hajek, 1998: 7).

En lo que al agua en específico concierne, el completo trabajo de Piwonka (1999) sobre el agua en Santiago entre los siglos XVI y XVIII es decidor de la riqueza interpretativa del elemento, a la luz de otros fenómenos sociales y problemas colindantes. En su obra se recorren los trazos coloniales de la organización y construcción de canales de regadío en las principales ciudades del país, pero también cuáles eran las instituciones que durante la conquista fueron clave en discutir políticamente sobre cómo gestionar el elemento.

4 Entiéndase por "régimen de naturaleza", siguiendo a Arturo Escobar, a las maneras específicas en que se desarrolla una relación entre el entorno natural y las dinámicas sociales en, o sobre, él desarrolladas. Escapando de un esencialismo dicotómico, que aleja la naturaleza de la cultura, la obra de Escobar propone encontrarse con puntos intermedios que denuncian la coconstrucción de los ambientes para la vida, los cuales son por cierto históricamente disputados. Para más detalle sobre la obra de Escobar, ver Gómez (2015). 
Asimismo, se arroja luces a las discusiones y reglamentos jurídicos que median la relación de la población con el agua. Todo ello es acompasado de una historia de la tecnificación y conquista de diversos cauces de agua que son el protagónico posibilitador de la urbanización a gran escala.

Sus limitaciones, junto con no avanzar más allá de 1740, están también por una aproximación más bien metropolitana de la revisión y por un enfoque aún muy reposado en la canalización como forma de existencia del agua en lo social. Tributando a este tremendo avance, así como también buscando actualizar las preocupaciones asociadas a lo hídrico en el panorama contemporáneo del país, es que a continuación el interés se centra en sujetos asociados a la gestión de agua más allá de la ciudad, pero sin enclaustrarse en el sujeto campesino, pues eso limitaría el movimiento meramente desde una canalización para el consumo a una para el riego. A contrapunto, se revisa en el presente texto cómo el proceso se acompasa de transformaciones y acumulaciones del mercado de aguas en Chile.

Otras formas de capturar al agua en la pregunta histórica son, como ya se ha mencionado, relacionándola con otras preocupaciones disciplinares y compartidas por las ciencias sociales. Llama la atención en esa agenda el trabajo colectivo de quienes presentan un abanico de posibles interrogantes sociales desde el agua (Roth et al, 2005), partiendo por la complejidad de los derechos de agua en clave jurídica, avanzando hacia las relaciones entre acceso al agua y políticas de equidad de género, la relación entre Estado y comunidades indígenas, enfrentado a la discusión sobre derechos inmemoriales como parte del legado colonial del proceso de construcción de los estados modernos.

Así vista, la preocupación histórica por la naturaleza ha sido parte de la tradición intelectual latinoamericana, particularmente teniendo como eje a las perspectivas asociadas a la ecología política. Esto se explica por la condición marcadamente antropogénica de los paisajes continentales, por un lado, así como por otro a los triunfos y derrotas que marcan la topografía latinoamericana en su intento por erigir ciudades, a pesar de las variadas condiciones y disponibilidades de ambientes para la vida en sociedad. Está siempre presente la condición de "conquistas a la naturaleza": atraviesa todo el territorio chileno y modela paisajes, actividades productivas, discursos sobre la modernidad y nuevos sujetos, mostrándose, así como una atractiva agenda de investigación muy fértil para el ejercicio interdisciplinar.

\section{Los sujetos del agua: ciudades y reforma agraria}

Uno de los agentes con los que más se vincula al agua en la producción historiográfica y sociológica es el campesinado. Esto ha devenido en una 
periodización de tales revisiones que toma como horizonte privilegiado a los años anteriores e inmediatamente posteriores al proceso de reforma agraria chilena. Tal producción historiográfica se ha ampliado a propósito de la reciente conmemoración del cincuentenario de su implementación (1968-2018), con miras a una lectura evaluativa sobre los aciertos y legados del proceso.

En la obra de Jacques Chonchol, por ejemplo, se observa cómo los sistemas agrarios en América Latina han sido construidos a lo largo del siglo XX a partir de efectos colaterales para poblaciones indígenas y consolidando una ruralidad basada en la figura del latifundio y el control de la propiedad. Cuando su trabajo se detiene en el caso chileno, observa cómo la violenta contrareforma orquestada en dictadura (1973-1989) ralentiza distribuciones de tierra en otras lógicas, junto con, además, propiciar la monopolización de derechos de agua y agudizar la condición especulativa de recursos naturales y lugares estratégicos para la atracción de capitales extranjeros (Chonchol, 2003: 13-19).

Coincide a grandes rasgos en el diagnóstico José Bengoa (2016), quien, desde una lectura histórico-antropológica, sostiene que, si bien el poder hacendal chileno ha experimentado situaciones de crisis y ruptura, en general ha gozado de una estabilidad y profundización claves en dar forma a los sujetos rurales del Chile central, sobre todo porque la figura del pequeño propietario, que podría contraponerse a esta estructura, es demasiado sensible a dificultades climáticas, con sus vaivenes productivos asociados, en general, y a los episodios de sequía, en particular.

Hernández (2006), por otro lado, tiende de manera directa el puente entre la evolución del marco jurídico del agua y el proceso de reforma agraria chileno. A su juicio, si bien la Reforma Agraria tiene como eje principal un cambio en las formas de propiedad de la tierra para el campesinado, hay una serie de procesos que acompañan este objetivo, que se relacionan con la pregunta por el aprovechamiento de los recursos hídricos (derechos de enajenación, nuevas normativas, entre otros). ${ }^{5}$

Desde otro enfoque, investigaciones sobre la Reforma Agraria argumentan que la estructura del agro en el Chile central es deudora protagónica de la forma en que el agua se ha gestionado y hecho disponible para el ejercicio de una serie de actividades productivas. En su revisión prima una lectura del agua como un elemento que, cuando controlado y tecnificado, cataliza el

5 Se puede pensar un proceso corto y largo de Reforma Agraria, si bien se frenó de golpe con la instalación de la dictadura militar el proceso de asignación de tierras, la derogación formal de la Reforma esperará hasta 1984 para realizarse. La mayor agitación política y campesina, a su vez, se rastrea en 1968, aunque las asignaciones y expropiaciones inician en 1965. 
progreso y conlleva modernización en sectores rurales. Así es como relata la transformación de un "árido" valle central a un "titánico" conjunto de empresas en estas tierras:

"La construcción de grandes obras de riego empieza con el Canal del Maipo en 1820, que transforma el árido secano que era, en el fértil valle que será 100 años después. El progreso del Valle Central lleva a mineros importantes del norte, como los Cousiño, los Subercaseaux, los Urmeneta, a adquirir grandes haciendo en el centro y dotarlas de regadío. Waddington conduce las aguas del río Aconcagua hasta Limache y Ramón Subercaseaux Mercado transforma los áridos suelos de Pirque al construir en 1834 el canal del mismo nombre. Una de las empresas titánicas es la obra del Canal de Las Mercedes, que tarda 30 años en terminarse, tiene un recorrido total de 120 kilómetros y comprende importantes obras de arte ( 3 túneles y un puente acueducto de 700 metros de largo sobre el río Puangue al llegar a Curacaví)" (Fontaine, 2001: 71).

Desde 1974, en Chile comienza a reforzarse la garantía y protección de la propiedad privada como condición clave del agua para ser transada en los mercados. Esto se consolidó en 1981, con la promulgación de la última modificación sustanciosa realizada a la fecha al Código de Aguas (vigente hasta 2018), que transforma las relaciones entre sociedad, agua y espacio mediante la promulgación de la disyunción entre tierra y agua, que permitía nuevos regímenes de propiedad, especulación y acumulación de derechos de aprovechamiento para usos privados más allá del consumo humano o riego vegetal, particularmente minería e hidroelectricidad.

Esta íntima relación entre la regulación del agua y el problema de la tierra, tópico protagonista en la revisión del proceso de reforma agraria chilena, queda capturada en la conclusión del trabajo de Hernández, cuando este sostiene que:

"La existencia de un mercado de aguas, por imperfecto y restringido que sea, ha impactado en la distribución de la propiedad agraria. El hecho que las personas puedan comprar, vender, dar en préstamo, arrendar y realizar cualquier negocio jurídico respecto del agua, incluyendo el decidir libremente su uso y su aplicación en cualquier predio o actividad ha facilitado el cambio de dueños de las tierras" (Hernández, 2006:14).

Este proceso fue acompañado tanto por la existencia del mercado que Hernández apunta, pero también a partir de una violenta descapitalización de la agricultura y las actividades asociativas del campo chileno. Según Reyes Coca (2014), por ejemplo, en la provincia de Nuble post Reforma Agraria la 
contracción de la agricultura respondió a la fuerza de las estructuras de propiedad históricas del agro nacional, donde la imposibilidad de los pequeños propietarios beneficiarios de la Reforma de competir con los antiguos dueños y su capitalización y tecnologización, rápidamente restituyó el orden de las cosas y fomentó una reconcentración de la propiedad de la tierra y el agua en las clases terratenientes.

Respecto del mismo espacio y horizonte temporal, Timmermann (2014) recoge la legitimación de la violencia y el miedo en la provincia de Ñuble, donde los efectos de la Reforma en el monopolio del poder político y cultural de las elites fueron rápidamente deslegitimados con una gran concentración de mecanismos del terror en la zona: comisarías, infantería, nuevas cárceles y episodios de reclusión masiva, tortura e interrogatorios acompañaron el proceso de dispersión de las ideas campesinas y tutelaron el proceso de reconcentración de tierras y aguas. Es este uno de los rostros de la estatalidad de las aguas, que se agudiza en su violencia durante dictadura y las acciones ejecutadas para retrotraer procesos políticos que mermaban la gobernabilidad de la Junta Militar. Sin embargo, esta violencia explícita y sistemática es luego reemplazada por otros mecanismos de legitimación de la lógica de mercado como respuesta frente a las contradicciones y dificultades asociadas a la forma que tomó el régimen de aguas chileno. Como se verá a continuación, son varios los rostros del Estado en el abordaje del agua como elemento para el ejercicio de su estatalidad en el país.

\section{Las estatalidades del agua en el Chile contemporáneo}

En ocasiones relegada a una condición secundaria de políticas sociales vinculadas al desarrollo urbano, la cobertura y acceso al agua, tanto potable como de riego, fue una preocupación a lo largo de todo el siglo XX chileno. De hecho, entre 1990 a 2013 se ha avanzado desde una cobertura efectiva del $67,7 \%$ al $94,4 \%{ }^{6}$ en lo que concierne al acceso a canalización y servicios higiénicos, y desde un $94,2 \%$ a un $99,2 \%$ en el mismo periodo en lo que respecta a la disponibilidad de agua potable.

Ya se ha mencionado cómo la literatura científica se ha concentrado en la canalización y disponibilidad de agua potable como una forma de analizar el hidropoder y la segregación socioespacial de las grandes ciudades. A continuación se mencionan brevemente cuáles son las demás estatali-

6 Número muy inferior al de la tasa de acceso a agua potable, particularmente explicado por la alta presencia en el país de campamentos, especialmente en Santiago y la región de Valparaíso, lo que robustece la hipótesis de la segregación socioespacial detrás del proceso de universalización del agua potable en Chile; ver Pflieger (2008). 
dades disponibles durante la segunda mitad del siglo XX en términos de gestión hídrica, y se identifica con ello cuáles son los organismos estatales que encarnan al objetivo. Estas estatalidades se solapan y desarrollan paralelamente, por lo que los momentos de esta tienen más que ver con su campo de acción que con hitos históricos específicos que delimiten una forma de otra.

El modelo neoliberal implementado durante la dictadura militar en Chile se hace manifiesto en la gestión del agua de consumo humano y, más específicamente, en lo que concierne a la distribución desigual de los accesos a agua potable en Chile, que además tiende a coincidir con la concentración de la riqueza en el Gran Santiago, luego de la aplicación de otros programas urbanísticos del régimen, tales como la "expulsión de pobres" (Hidalgo et al, 2016) y reorganización de los límites comunales en la Región Metropolitana. Lo anterior se puede observar en el siguiente cuadro comparativo desarrollado por Pflieger (2008), en el que se exhibe, a la izquierda, las comunas con mayor acceso a agua potable y, a la derecha, los ingresos per cápita por comuna.

Figura 1

Tasas de acceso a la vivienda y alcantarillado en el Gran Santiago, 1982

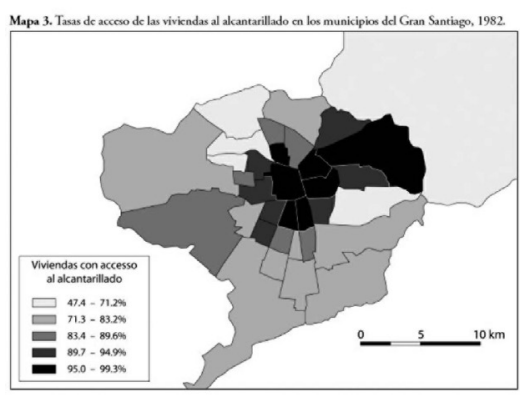

Fuenter Elabonación propia

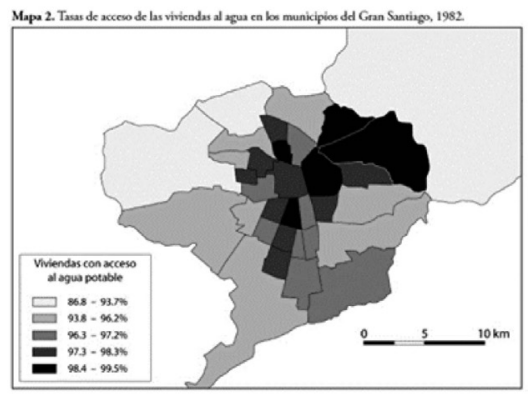

Fuenter Elaboración propia.

Fuente: Pflieger, 2008.

En primer lugar, se observa un interés estatizante de legitimar la segmentación de la ciudad con cargo a criterios técnicos e higiénicos, a propósito de la canalización del agua como una conquista de la modernidad frente a la vida en sociedad. En este campo de acción y discursos emerge como agente clave de la estatalidad la figura del Municipio (Pflieger, 2008). Durante el periodo 1950-1975 el discurso de la universalización del agua potable sirvió como moneda de cambio para referirse al progreso de la nación, que se buscó asociar rápidamente a las políticas de vivienda y a la ejecución de programas por parte del Ministerio de Obras Públicas, y a las políticas de planificación urbanas desde Allende a Pinochet, décadas en las que se concentra el creci- 
miento sustantivo de las tasas de acceso al agua potable en Chile (Pflieger, 2008 y Pérez et al., 2005).

Resuelto el problema de la canalización e instalada la noción de un país moderno con ciudades integradas, la neoliberalización del agua vehiculizada por la promulgación del Código de Aguas en dictadura (1981) invita a una estatalidad promotora del mercado, cuyo principal actor es ahora la Dirección General de Aguas (DGA). Este movimiento de organismos es sintomático de un desplazamiento de preocupaciones estatales: desde el agua como una política de redes e integración hacia una política de mercado sectorizado. En general, el mercado de agua ofrece pocos movimientos como tal y se ha enfocado en Chile a la concentración de Derechos de Agua para usos de especulación y no como parte del ideario de activación del agro. De hecho, la transacción de derechos en pequeños terrenos agrícolas es casi nula, mientras que los volúmenes de inversión en cuencas cercanas a yacimientos mineros y sectores de fruticultura intensiva con especies extranjeras solo ha ido en aumento desde 1985 a la fecha (Bauer, 2002: 89).

En un tercer momento se puede identificar un giro hacia la conservación del patrimonio ambiental en general y del agua en particular, promovido por agentes no estatales, tales como organizaciones internacionales, ONG locales y academia, que redunda en una nueva matriz discursiva del Estado articulada en torno al Sistema Nacional de Áreas Silvestres Protegidas por el Estado (SNASPE). En este momento la estatalidad es moralizante y busca concientizar respecto de la importancia de la preservación del patrimonio ambiental, que es entendido como simultáneamente rico por sí mismo, pero también a propósito del potencial turístico, promovido desde SERNATUR, dependiente del Ministerio de Economía, que vendría a operar como una actualización de las formas más masivas de interés en el capital extranjero. En este momento aparece la filantropía empresarial, vinculada a la protección de grandes extensiones de terreno con acceso a fuentes estratégicas de agua en el sur de Chile (Holmes, 2014; Orrego, 2011).

En esta revisión de las estatalidades del agua aparece un agente como móvil a lo largo del tiempo. Esto evidentemente tiene relación con cómo la estatalidad es dinámica y en su despliegue organiza y reorganiza componentes de la gobernabilidad según determinadas agendas políticas. ${ }^{7}$ Particularmente esto se refiere, para el caso del agua, a CONAF, organismo que ha cumplido un rol anfibio en la gestión productiva y de conservación del patrimonio ambiental del país.

Ver, por ejemplo, el trabajo de Puente (2015), en el que señala que los archivos campesinos del Perú sufren de la misma dispersión que el tratamiento constitucional que el país tiene para con sus pueblos indígenas a lo largo del siglo XX. 
Es así como durante la década de 1950, la Corporación Nacional Forestal (organismo privado con alcance público en la ejecución de políticas forestales), actuó como un activo promotor de la gestión privada de los bosques, basada en la alarma de la desertificación para el poblamiento con especies que luego darían pie al boom de la fruticultura en el Valle Central (véase, por ejemplo, el stress hídrico al que se ve sometido el Valle de Limarí en Budds, 2008) o a los esfuerzos de la Sociedad Nacional de Agricultura por activar económicamente la zona desde mediados del siglo XIX en adelante (Lacoste et al., 2013). En un segundo momento sirvieron a la estatalidad promotora del capital extranjero mediante su protagónico rol en la gestión y defensa del sentido del Decreto con Fuerza de Ley 701, que regulaba el fomento a las plantaciones forestales con un subsidio de $75 \%$ a empresarios del rubro y que, a la fecha (2018), sigue vigente y es signado como uno de los elementos del marco jurídico exportador de commodity más perjudicial para la biodiversidad del país. Finalmente, y acompasándose con una nueva estatalidad basada en el discurso de la conservación y la turistificación del patrimonio ambiental, durante las décadas de 2000 y 2010 actuó como organismo encargado de la gestión del Servicio Nacional de Áreas Silvestres Protegidas y recientemente, desde 2014, como promotor de la Ley de Conservación del Bosque nativo (Moreno, 2015).

Figura 2

Resumen de la tipología de estatalidades, agentes estatales protagonistas y discursos

\begin{tabular}{|l|l|l|l|}
\hline Estatalidad & \multicolumn{1}{|c|}{ Periodo } & $\begin{array}{c}\text { Agente } \\
\text { precursor }\end{array}$ & \multicolumn{1}{c|}{ Campo discursivo } \\
\hline $\begin{array}{l}\text { Control y } \\
\text { Segregación }\end{array}$ & $1975-1985$ & $\begin{array}{l}\text { MOP } \\
\text { Municipio } \\
\text { MINAGRI }\end{array}$ & $\begin{array}{l}\text { La canalización y llegada de nuevas } \\
\text { tecnologías para la gestión hídrica, } \\
\text { como indicador inequívoco del } \\
\text { progreso del país y de los beneficios de } \\
\text { la expansión urbana para las ciudades, } \\
\text { y de la tecnificación de las actividades } \\
\text { agropecuarias para el campo. }\end{array}$ \\
\hline $\begin{array}{l}\text { Promotora } \\
\text { mercantil }\end{array}$ & 1981 -presente & DGA & $\begin{array}{l}\text { La necesidad de desregular la } \\
\text { compra y venta de derechos de } \\
\text { agua para estimular el crecimiento } \\
\text { y el emprendimiento de pequeños } \\
\text { productores agropecuarios. }\end{array}$ \\
\hline Moralizante & $2000-2014$ & $\begin{array}{l}\text { SNASPE } \\
\text { SERNATUR }\end{array}$ & $\begin{array}{l}\text { La necesidad de concientizar a } \\
\text { la población de la importancia } \\
\text { de las acciones individuales y de } \\
\text { la conservación museológica del } \\
\text { patrimonio ambiental con acceso a } \\
\text { cuencas hídricas clave. }\end{array}$ \\
\hline
\end{tabular}

Fuente: Elaboración propia. 
Como se aprecia en la figura 2, en Chile las estatalidades en torno a la gestión de agua están en un zigzagueo constante, que distingue entre medidas promotoras de la desregulación en el mercado del recurso, por un lado, y la conciencia y responsabilidad individual en el cuidado del patrimonio ambiental posibilitado por el agua, por otro.

Asimismo, es posible identificar una narrativa del desarrollo que encuentra su apoyo en los proyectos hidráulicos. Es decidor sobre ello, en un sentido de autobiografía institucional, la forma en que los servicios sanitarios en Chile han comprendido y socializado su rol en el país. De esta manera, cuando se reconstruyen los primeros hitos de canalización y servicios de gestión de agua para consumo humano, la tarea se aborda con un sentido modernizador que es transversal y que luego permitirá asociarse a la privatización de estos servicios:

"A mediados del siglo XIX, Chile se incorporó a los tiempos modernos, al construirse algunas importantes obras de ingeniería sanitaria como: la primera cañería surtidora de agua potable para la ciudad de Valparaíso, en 1850" (Historia del Sector Sanitario en Chile, 2018).

En la misma publicación, de hecho, se manifiesta la alta tasa de privatización de los servicios de canalización y aguas servidas en Chile, a partir de una reforma realizada en dictadura, el año 1989. Antes de ello, la provisión de cañerías se repartía entre privados, municipios y un gran porcentaje capturado por el Servicio Nacional de Obras Sanitarias (SENDOS).

\section{Figura 3}

Resumen de distribución de prestadores sanitarios en chile, 1989-2005

\begin{tabular}{|l|c|c|}
\hline Año & Concesión operada por privados & Municipal, cooperativas o estatal \\
\hline 1989 & $3,8 \%$ & $96,2 \%$ \\
\hline 2005 & $95,4 \%$ & $4,6 \%$ \\
\hline
\end{tabular}

Fuente: Elaboración propia, con base en información de la Superintendencia de Obras Sanitarias, 2019.

\section{Conclusiones}

"I argue here that water was much more central to the formation and effectiveness of the wider neoliberal programme in Chile, and to the consolidation of elite power and corporate alliances under both dictatorship and democracy, than hitherto acknowledged. Second, starting from the critique that many analyses overattribute outcomes to neoliberal policies per se, and underplay the ideologies and motives 
of their proponents, I suggest that previous scholarship has largely focused on formal state politics and has paid insufficient attention to the roles of specific interest groups, especially technocrats, and their political alliances and strategic ambitions, within the neoliberalisation of nature. Third, I build on Swyngedouw's analysis of hydraulic engineering to show how water reforms can also recast and consolidate power relationships, and produce waterscapes with distinct characteristics" (Budds, 2013: 247-266).

El párrafo anterior forma parte del programa de investigación de una geografía crítica e histórica de Jessica Budds respecto del Valle Central chileno, específicamente en lo que concierne a Petorca. De su carta de navegación emergen tres puntos que coinciden con la manera en que a lo largo del texto esta investigación se ha buscado aproximar a los problemas que, desde el agua, se pueden observar en la construcción del Chile contemporáneo. A saber:

i) La consolidación de las fuerzas corporativas de la elite en dictadura y en transición, en colaboración constante con una estatalidad permisiva de la acumulación como nueva forma de relacionarse con los recursos naturales.

ii) Un interés en el entramado tecnopolítico detrás de las transformaciones neoliberalizadoras, normalmente ocultas detrás de los sujetos y lugares predilectos del interés académico sobre las transformaciones del agro chileno.

iii) Lo anterior decanta en la producción de nuevos paisajes del agua, posibilitados por la forma en que determinados agentes hacen uso del poder hidráulico que este entramado les confiere.

Es así como esta inmersión tentativa a las formas en que se ha investigado sobre las aguas en Chile arroja luces sobre líneas de profundización de interés para reflexionar sobre otros agentes vinculados a la gestión del agua, siempre buscando salir de la práctica concreta de lo agro hacia los demás campos, como pueden ser los gremios profesionales de la sanidad, de la conservación, pero también de la patrimonialización y monumentalidad de la naturaleza.

En las emociones y otras nuevas entradas metodológicas al problema aparece también un fértil camino por acompañar, en el que las topofilias, pero también los impactos de los discursos estatalizadores son manifestados por los agentes, modificando así prácticas y memorias sobre el agua como un bien del progreso de la nación, de consumo en el mercado, o frágil y urgente de conservar. Estas narrativas asociadas a agentes estatales y tipos de moralidad y formas de comportamiento observadas también pueden ser indicador para una posterior consulta a fuentes de la época, que estén entroncadas a diversos intereses y organismos técnicos, productivos, científicos y ciudadanos, para lograr tener una visión de conjunto respecto al cómo se 
construyen, median y disputan las formas de comprender y sentir sobre el agua en el Chile contemporáneo.

Contemporaneidad que se aprecia en el agua como plataforma de disputa y de privilegiada observación a las contradicciones promovidas por el proceso de neoliberalización. Dentro de las cuales vale la pena mencionar, como ya se ha insinuado a lo largo del artículo: (i) las transformaciones en la matriz energética a lo largo de la modernización capitalista en Chile; (ii) la producción de una nueva ruralidad dependiente de una economía de servicios turistificada, y (iii) los conflictos étnicoterritoriales asociados al recurso y que ponen en tensión el proyecto y campo discursivo de la unidad nacional. Aspectos todos rastreables desde la tipología de estatalidades mencionadas, efectuadas desde agentes concretos con soportes identificables.

Así, finalmente, sobre el enfoque "lo ambiental" se vislumbra su doble vocación para con el agua como objeto fijo, pero también como construcción. La tríada inicial mencionada: material, inmaterial y biopolítica, es una invitación a complejizar las herramientas y perspectivas mediante las cuales se discute desde, y no solo acerca de lo medioambiental como parte del problema de lo contemporáneo.

\section{Referencias bibliográficas}

\section{Fuentes primarias}

a) Archivos

Archivo de la Comisión Nacional de Energía, Chile.

b) Bases de datos y repositorios institucionales

Superintendencia de Servicios Sanitarios, Chile. Disponible en: http://www. siss.gob.cl

Dirección General de Aguas. Disponible en: http://www.dga.cl

Historia del Sector Sanitario en Chile (2018). Disponible en: http://www.siss. gob.cl/586/w3-article-3681.html, revisado el 20-XI-2018

\section{Fuentes secundarias}

a) Artículos y capítulos de libros

Bakker, K. (2012). "Water: Political, biopolitical, material", en Social Studies of Science, Vol. 42, N 4, pp. 616-623. 
Budds, J. (2008). "Whose scarcity? The hydrosocial cycle and then changing waterscape of La Ligua basin, Chile", en M. Goodman, M. Boykoff y K. Evered (Editores), Contentious Geographies: Environment, Meaning, Scale. Aldershot: Ashgate, pp. 59-68.

Budds, J. (2013). "Water, Power, and the Production of Neoliberalism in Chile, 1973-2005", en Environment and Planning D: Society and Space, Vol. 31, № 2, pp. 301-318.

Bustos, B. y F. Irarrázaval (2016). "'Throwing Money into the Sea': Capitalism as a World ecological System. Evidence from the Chilean Salmon Industry Crisis, 2008", en Capitalism Nature Socialism, Vol. 26, № 3, pp. 83-102.

Castro, J. y M. Lavinas (2017). "Stateness as Landgrab: A political History of Maya Dispossession In Guatemala", en American Quarterly, Vol. 69, № 4, pp. 791-799.

Chonchol, J. (2003). "La reforma agraria en América Latina", en CIDES-UMSA (Editores), Proceso agrario en Bolivia y América Latina. La Paz: Plural Editores, pp. 205-222.

Cowan, C. (2016). "Estatalidades, políticas públicas y movimientos sociales en su configuración interdependiente. Una perspectiva analítica", en Contested_Cities. Working Paper Series, disponible en http://contested-cities.net/working-papers/ wp-content/uploads/sites/8/2017/05/WPCC_160007_Estado_final.pdf, revisado el 10-XI-2018.

Hidalgo, R., V. Alvarado y D. Santana (2016). "Los expulsados de la metrópoli: expolio y esquilmo en la locación de la vivienda social en la ciudad neoliberal. Una perspectiva de Santiago y Valparaíso", en Estudios Socioterritoriales, Nㅜ 20, pp. 41-55.

Gaynor, A. (2017a). "Lawnscaping Perth: Water Supply, Gardens and Scarcity, 1890-1925", en Journal of Urban History, disponible en https://doi. org/10.1177/0096144217692991, revisado el 10-XI-2018.

Gaynor, A. (2017b). "Self-sown crops, modernity and the making of mallee agricultural landscapes", en Agricultural History, Vol. 91, № 2, pp. 171-186.

Gaynor, A. y J. McCann (2017). "I've had dolphins ... looking for abalone for me': Oral history and subjectivities of marine engagement", en The Oral History Review, Vol. 4, № 2, pp. 260-277.

Gómez, M. (2015). "Una aproximación a las contribuciones de Arturo Escobar a la Ecología Política", en Revista de Ecología Política, № 50, pp. 100-105.

Hernández, R. (2006). "Reforma Agraria y Evolución del Marco Jurídico del Agua en Chile" en Serie de Estudios Legislativos, FAO.

Holmes, G. (2014). "What is a land grab? Exploring green grabs, conservation, and private protected areas in southern Chile", en Journal of Peasant Studies, Vol. 41, No 4, pp. 547-567. 
Lacoste, P., M. Aranda, J. A. Yuri, A. Castro, A. Garrido y B. Rendón (2013). "La Sociedad Nacional de Agricultura (SNA) y el desarrollo de la fruticultura en Chile, 1838-1933", en Mundo Agrario, Vol. 13, № 26, pp. 1-23.

Pérez Oyarzun, F., J. Rosas y L. Valenzuela (2005). "Las aguas del centenario", en $A R Q$ (Santiago), N 60, pp. 72-74.

Pflieger, G. (2008) "Water and power in Santiago de Chile: Socio-spatial segregation through network integration", en Geoforum, Vol. 39, № 6, pp. 1907-1921.

Puente, J. (2015). "Archivos Campesinos: San Juan de Ondores, Actas Comunales e Historias Rurales en el Perú, 1937-2012", en C. Aguirre y J. Villa Flores (Editores), From the Ashes of History: Loss and Recovery of Archives and Libraries in Modern Latin America. Raleigh: North Carolina State University y A Contracorriente Press, pp. 267-306.

Reyes Coca, M. (2014). "La descapitalización de la agricultura de Ñuble: 18741980", en Tiempo y Espacio, № 9, pp. 41-51.

Scheer, P. (2012). "Are Emotions a Kind of Practice (and is That What Makes Them Have a History)? A Bourdieuian Approach to Understanding Emotion", en History and Theory, № 51, pp. 193-220.

Skewes, J., y F. Trujillo y D. Guerra (2017). "Transformaciones de los modos de significar el espacio habitado", en Revista INVI, Vol. 32, № 91, pp. 23-64.

Swyngedouw, E. (2007). "Techno natural revolutions. The scalar politics of Franco's hydro-social dream for Spain, 1939-1975", en Transactions, Institute of British Geographers, Vol. 32, № 1, pp. 9-28.

Timmermann, F. (2014). "Legitimación, violencia y miedo en la provincia de Nuble. Régimen cívico-militar, 1973", en Tiempo y Espacio, № 28, pp. 183-206.

\section{b) Libros}

Bauer, C. (2002). Contra la corriente: Privatización, mercados de agua y el Estado en Chile. Santiago: LOM Ediciones.

Bengoa, J. (2016). Historia rural del Chile Central. Dos tomos. Santiago de Chile: LOM Ediciones.

Camus, P. (2005). Ambiente, bosques y gestión forestal en Chile. 1541-2001. Santiago de Chile: LOM Ediciones.

Camus, P. y E. Hayek (1998). Historia ambiental de Chile. Santiago de Chile: Andros Editores.

Fontaine Aldunate, A. (2001). La tierra y el poder. Reforma Agraria en Chile (1964-1973). Santiago: Zig-Zag. 
Gárate, M. (2012). La revolución capitalista de Chile (1973-2003). Santiago de Chile: Ediciones Universidad Alberto Hurtado.

Myllyntaus, T. y M. Saikku (1999). Encountering the Past in Nature: Essays in Environmental History. Athens: Ohio University Press.

O'Donnell, G. (2010). Democracia, agencia, estado: Teoría con intención comparativa. Buenos Aires: Prometeo Libros.

Orrego, J. (2011). La entropía del capitalismo. Santiago de Chile: Ecosistemas Ediciones

Piwonka, G. (1999). Las aguas de Santiago de Chile. 1541-1999. Tomo I. Los primeros doscientos años. 1541-1741. Santiago: Centro de Investigaciones Barros Arana.

Programa de las Naciones Unidas para el Medio Ambiente (2000). GEO América Latina y el Caribe. Perspectivas del Medio Ambiente. Costa Rica: PNUMA.

Roth, D., R. Boelens y M. Zwarteveen (2005). Liquid relations. Contested water rights and Legal complexity. New Jersey: Rutgers University Press.

Sojo, C. (2011). The State under Scrutiny: Public opinion, Stateness and government performance in Latin America. Santiago de Chile: Naciones Unidas Ediciones.

Tortolero, A. (2000). El agua y su historia: México y sus desafíos hacia el siglo XXI. Buenos Aires: Siglo XXI Editores.

c) Tesis

Moreno, F. (2015). Análisis Histórico Jurídico de la legislación del bosque nativo en Chile. Universidad de Chile: Tesis para optar al grado académico de Licenciado en Ciencias Jurídicas, Facultad de Derecho. 